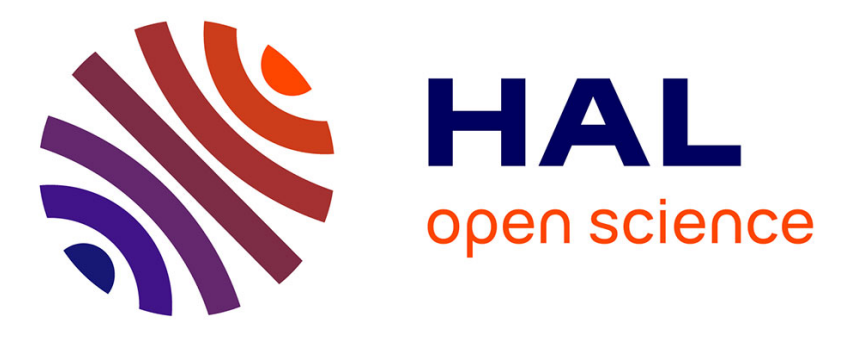

\title{
New Spins for ground states and isomers in ${ }^{115} \mathrm{Pd}$ and ${ }^{117} \mathbf{P d}$
}

W. Urban, A. Zlomaniec, G.S. Simpson, J.A. Pinston, J. Kurpeta, T. Rzaca-Urban, J.L. Durell, A.G. Smith, B.J. Varley, N. Schulz, et al.

\section{- To cite this version:}

W. Urban, A. Zlomaniec, G.S. Simpson, J.A. Pinston, J. Kurpeta, et al.. New Spins for ground states and isomers in ${ }^{115} \mathrm{Pd}$ and ${ }^{117} \mathrm{Pd}$. European Physical Journal A, 2004, 22, pp.157-161. 10.1140/epja/i2004-10090-0 . in2p3-00023331

HAL Id: in2p3-00023331

https://hal.in2p3.fr/in2p3-00023331

Submitted on 23 Nov 2004

HAL is a multi-disciplinary open access archive for the deposit and dissemination of scientific research documents, whether they are published or not. The documents may come from teaching and research institutions in France or abroad, or from public or private research centers.
L'archive ouverte pluridisciplinaire $\mathbf{H A L}$, est destinée au dépôt et à la diffusion de documents scientifiques de niveau recherche, publiés ou non, émanant des établissements d'enseignement et de recherche français ou étrangers, des laboratoires publics ou privés. 


\title{
New spins for ground states and isomers in ${ }^{115} \mathbf{P d}$ and ${ }^{117} \mathbf{P d}$
}

\author{
W. Urban ${ }^{1}$, A.Złomaniec ${ }^{1}$, G. Simpson ${ }^{2}$, J.A. Pinston ${ }^{3}$, J. Kurpeta ${ }^{1}$, T. Rzạca-Urban ${ }^{1}$, J.L. Durell ${ }^{4}$, A.G. Smith ${ }^{4}$, \\ B..J. Varley ${ }^{4}$, N. Schulz ${ }^{5}$, and I. Ahmad ${ }^{6}$ \\ 1 Faculty of Physics, Warsaw University, ul.Hoża 69, 00-681 Warsaw, Poland \\ 2 Institut Laue-Langevin, F-38042 Grenoble Cedex, France \\ 3 Laboratoire de Physique Subatomique et de Cosmologie, IN2P3-CNRS/Université J. Fourier, F-38026 Grenoble Cedex, France \\ 4 Schuster Laboratory, Department of Physics and Astronomy, University of Manchester, Manchester M13 9PL, UK \\ 5 Institut de Recherches Subatomiques UMR7500, CNRS-IN2P3 et Université Louis Pasteur, 67037 Strasbourg, France \\ 6 Argonne National Laboratory, Argonne, IL 60439, USA
}

\begin{abstract}
Abstact Levels in ${ }^{115} \mathrm{Pd}$ and ${ }^{117} \mathrm{Pd}$ nuclei, populated in the spontaneous fission of ${ }^{248} \mathrm{Cm}$ were studied by means of prompt gamma spectroscopy using the EUROGAM2 array of Anti-Compton spectrometers. Negative-parity, I=9/2 excitations were identified, which are associated with the long-lived isomers in these nuclei, reported previously as $11 / 2^{-}$excitations. The new data indicate spin and parity $3 / 2^{+}$for ground states in ${ }^{115} \mathrm{Pd}$ and ${ }^{117} \mathrm{Pd}$ instead of $5 / 2^{+}$proposed in previous works. This result implicates changes of spin assignments to other levels in both nuclei.
\end{abstract}

PACS. : 21.10. -k, 21.10.Re, 21.10.Tg, 23.20.Lv, 23.20. -g, 23.20.En, 25.85.Ca, 27.60.+j

The $\nu \mathrm{h}_{11 / 2}$ neutron shell plays a crucial role in defining patterns of the near-yrast excitations in the neutron-rich nuclei of the $100<\mathrm{A}<120$ region. It has been shown, that population of the $1 / 2^{-}[550]$ and $3 / 2^{-}$[541] orbitals originating from the $\mathrm{h}_{11 / 2}$ neutron shell is responsible for the onset of deformation in the mass $A \sim 100$ nuclei at $N \sim 60$ [1-4]. At neutron numbers $N \geq 61$ deformed bands, based on the $5 / 2^{-}[532]$ orbital are observed, corresponding to prolate shape [5-9]. An interesting question is how the $\nu \mathrm{h}_{11 / 2}$ shell influences the nuclear structure at still higher neutron numbers.

In a recent theoretical study [10] of neutron-rich nuclei from the $A \sim 110$ region it has been suggested that in these nuclei, where the Fermi level of both, protons and neutrons approaches high-energy subshells, one may expect oblate deformation in ground states. However, experimental studies of neutron-rich palladium isotopes report that the deformation is prolate up to the neutron number $N=72,[11,12]$. There are also calculations, which do not reveal any oblate shape in this region. Instead, a weak prolate deformation, slowly decreasing to zero when approaching the $\mathrm{N}=82$ shell, is reported [12]. More data is needed to clear this discrepancy. One can see in the single-particle diagrams shown in Ref.[10] that there is a clear difference between excitation patterns corresponding to prolate and oblate deformation. Therefore, a detailed knowledge of single-particle excitation patterns in odd-A nuclei from this region might be helpful. However, while the $\Omega \leq 5 / 2$ orbitals of the $\nu \mathrm{h}_{11 / 2}$ parentage are well documented, the high- $\Omega \nu \mathrm{h}_{11 / 2}$ subshells are less known. The identification of high- $\Omega$ configurations in this region becomes, therefore, an important task.

A general picture of yrast excitations in the neutronrich ${ }^{115} \mathrm{Pd}$ and ${ }^{117} \mathrm{Pd}$ nuclei, proposed in recent studies [11-13], is that of a decoupled rotational band on top of the $\nu \mathrm{h}_{11 / 2}$ orbital, with weakly deformed, low-lying $11 / 2^{-}$ band head forming a long-lived isomer, due to a large spin difference between the band head and the $5 / 2^{+}$ground state. This is quite different from the situation observed in neutron-rich ruthenium isotopes, just two protons below, where negative-parity bands are based on the $5 / 2^{-}$ and $7 / 2^{-}$orbitals originating from the $\nu \mathrm{h}_{11 / 2}$ shell and where long-lived isomers are not formed. One may try to attribute this difference to the proximity of the $Z=50$ closed shell, where less deformed and spherical, high- $\Omega$ orbitals are expected. We note, however, that the $394.8 \mathrm{keV}$, $15 / 2^{-} \rightarrow 11 / 2^{-}$transition in ${ }^{115} \mathrm{Pd}$ has in fact lower energy than the corresponding $415.9 \mathrm{keV}$ transition in ${ }^{113} \mathrm{Ru}$. This suggests that ${ }^{115} \mathrm{Pd}$ is deformed and the $\nu \mathrm{h}_{11 / 2}$ shell should split into subshells in this nucleus.

There are hints that $9 / 2^{-}$(and possibly $7 / 2^{-}$) orbitals may be present in ${ }^{115} \mathrm{Pd}$ and ${ }^{117} \mathrm{Pd}$ isotopes at low excitations. Penttilä et al $[14,15]$ found that the $T_{1 / 2}=0.3 \mathrm{~s}$ isomer at $81 \mathrm{keV}$ in ${ }^{113} \mathrm{Pd}$ has spin $9 / 2^{-}$. This result has been confirmed recently by Houry et al [12], who reported rotational bands on top of both, the $9 / 2^{-}$and the $11 / 2^{-}$ levels in ${ }^{113} \mathrm{Pd}$. The bands are connected by the $407 \mathrm{keV}$ transition, which fixes the position of the $11 / 2^{-}$level at $18 \mathrm{keV}$ above the $9 / 2^{-}$level. The $9 / 2^{-}$is also known in ${ }^{109} \mathrm{Pd}$ where it is placed $98 \mathrm{keV}$ above the $11 / 2^{-}$isomer [16]. A simple extrapolation based on these two cases 


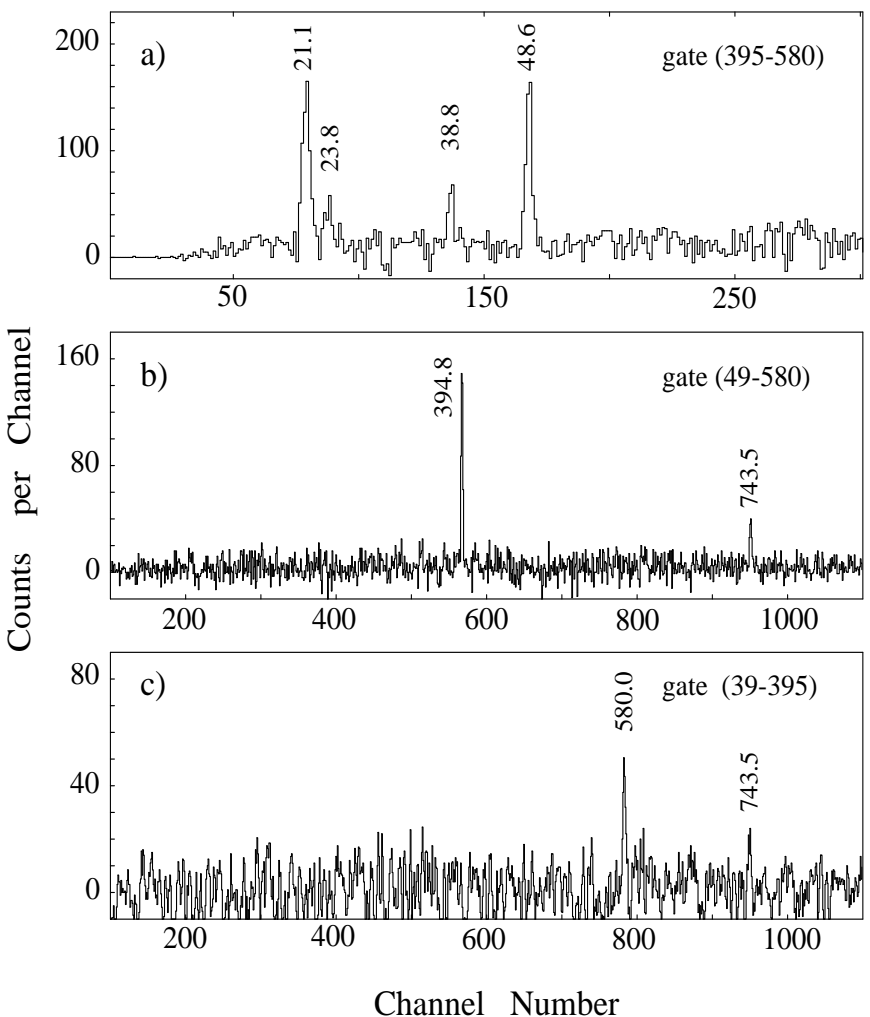

Fig. 1. $\gamma-\gamma$ coincidence spectra double-gated on lines in ${ }^{115} \mathrm{Pd}$. Transition energies and gates are given in $\mathrm{keV}$.

suggests that in ${ }^{115} \mathrm{Pd}$ and ${ }^{117} \mathrm{Pd}$ the $9 / 2^{-}$level may be located below the $11 / 2^{-}$level and, consequently, may correspond to isomeric levels in these nuclei. This, in turn, would imply changes of ground-state spins there.

In this work we report on the identification of $9 / 2^{-}$ excitations in the ${ }^{115} \mathrm{Pd}$ and ${ }^{117} \mathrm{Pd}$ nuclei. In our study we used multiple-gamma coincidence data from a measurement of prompt gamma radiation following spontaneous fission of ${ }^{248} \mathrm{Cm}$. High-fold coincidences between promptgamma rays were measured using the EUROGAM2 array [17] plus Low Energy Photon (LEP) detectors. The data were converted into triple coincidences (providing about $2 \times 10^{10}$ events) and sorted into various three-dimensional histograms. More details on the experiment and the data analysis can be found in Refs. [4, 18-20].

We have searched for levels in the vicinity of the $11 / 2^{-}$ excitation in ${ }^{115} \mathrm{Pd}$, reported as $\mathrm{T}_{1 / 2}=50 \mathrm{~s}$ isomer at 89.3 $\mathrm{keV}$ [21]. To look for low energy gamma lines we used triple coincidences where one of the energies was measured by the LEP detector and the other two by Ge detectors of the EUROGAM. In Fig. 1a a low-energy part of the LEP spectrum, double gated on the $394.8 \mathrm{keV}$ and $580.0 \mathrm{keV}$ lines from the negative-parity band in ${ }^{115} \mathrm{Pd}$, is shown. Clearly seen is a line at $48.6 \mathrm{keV}$ and palladium $K_{\alpha}$ and $K_{\beta} \mathrm{X}$-ray lines at $21.1 \mathrm{keV}$ and $23.8 \mathrm{keV}$, respectively. In Fig.1b we show a spectrum doubly gated on the $48.6 \mathrm{keV}$ and $580.0 \mathrm{keV}$ lines. One can see the $394.8 \mathrm{keV}$ and 743.5 $\mathrm{keV}$ lines of the negative-parity band in ${ }^{115} \mathrm{Pd}$. Intensities seen in Fig.1 and other gated spectra indicate that the

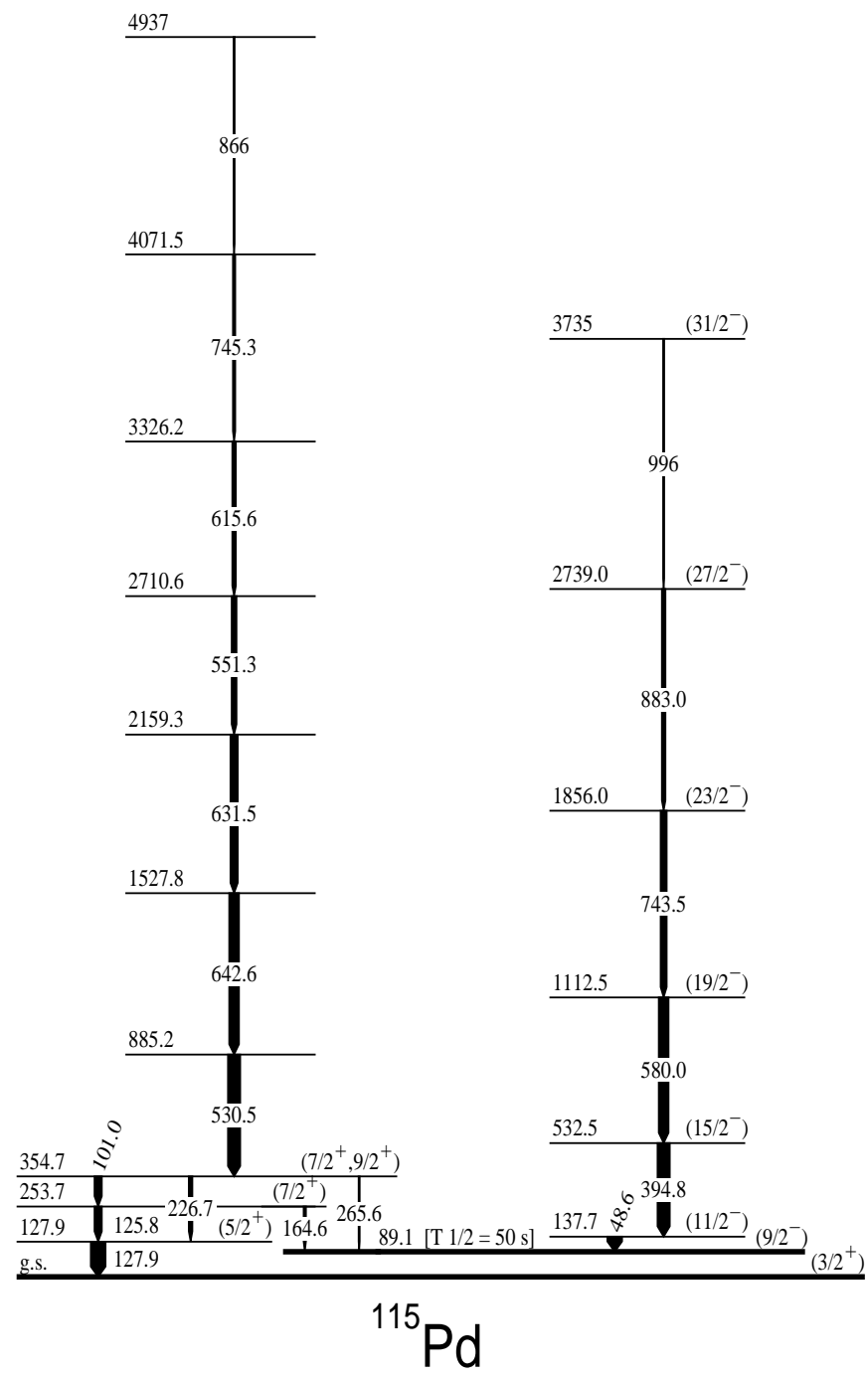

Fig. 2. Partial decay scheme of ${ }^{115} \mathrm{Pd}$, as obtained in this work.

$48.6 \mathrm{keV}$ transition is located in the negative-parity band below the $394.8 \mathrm{keV}$ line rather than somewhere higher in the band.

In Fig.1a one can also see a line at $38.8 \mathrm{keV}$, which is in coincidence with the $394.8 \mathrm{keV}, 580.0 \mathrm{keV}$ and 743.5 $\mathrm{keV}$ lines, as illustrated in Fig.1c, showing a spectrum double gated on the $38.8 \mathrm{keV}$ and $394.8 \mathrm{keV}$ lines. The $38.8 \mathrm{keV}$ line is most likely located at the the bottom of negative-parity cascade in ${ }^{115} \mathrm{Pd}$, as suggested by the intensities in the coincidence spectra. We could not see any coincidences between the $48.6 \mathrm{keV}$ and the $38.8 \mathrm{keV}$ lines. Therefore, we could not establish, with certainty, the position of the $38.8 \mathrm{keV}$ relative to the $48.6 \mathrm{keV}$ transition. This negative result is probably due to an experimental limit and a better measurement may resolve the problem.

From Fig. 1a one can estimate conversion coefficients for the $38.8 \mathrm{keV}$ and $48.6 \mathrm{keV}$ transitions assuming that the X-ray intensity, seen in Fig.1a, is due to the conversion of the two lines. The theoretical values are 1.0 for E1, 2.6 for M1 and 10.3 for an E2 transition at $49 \mathrm{keV}$ and 1.9 for E1, 5.0 for M1 and 18.5 for an E2 transition at $39 \mathrm{keV}$ (we 
did not consider M2 multipolarity due to the prompt character of both transitions ). Taking the efficiency-corrected, relative intensities of the palladium $\mathrm{K}_{\alpha}$ line at $21.2 \mathrm{keV}$, the $38.8 \mathrm{keV}$ line and $48.6 \mathrm{keV}$ line we conclude that it is not possible that both lines have an E1 multipolarity. If one of the lines is an E1 then the other is M1+E2 with the resulting conversion coefficient of $8(2)$ for the $48.6 \mathrm{keV}$ line or $16(4)$ for the $38.8 \mathrm{keV}$ line (both values being close to a limit expected for an E2 multipolarity). The other possibility is that both transitions are of a M1+E2 character. If, for instance, they have the same intensity (are in a cascade) their conversion coefficients are $9(2)$ and 5(1) for the $38.8 \mathrm{keV}$ and $48.6 \mathrm{keV}$ lines, respectively, both consistent with a M1+E2 multipolarity. The above results leave several solutions. Fortunately, there are other constraints:

i) there is only one long-lived isomer observed in ${ }^{115} \mathrm{Pd}$ and the isomeric transition is an E3, as deduced from its conversion coefficient [21]. The $\mathrm{T}_{1 / 2}=50 \mathrm{~s}$ half life of the isomer implies a high hindrance of 497 for this E3 transition [21]. Because of this long half-life one might consider a $\Delta \mathrm{I}=4$ character of the isomeric transition. We note, however, that the decay of the $253.7 \mathrm{keV}$ level to the $89.1 \mathrm{keV}$ isomer by the $164.6 \mathrm{keV}$ transition [22] on one hand and to the ground state level by a cascade of the $125.8 \mathrm{keV}$ and $127.9 \mathrm{keV}$ transitions, both of $\mathrm{M} 1+\mathrm{E} 2, \Delta \mathrm{I}<1$ character $[14,22]$, on the other hand, excludes the $\Delta \mathrm{I}=4$ character of the $89.3 \mathrm{keV}$ isomeric transition;

ii) while the $9 / 2^{-}$excitation in ${ }^{115} \mathrm{Pd}$ may be easily explained, as due to $\mathrm{h}_{11 / 2}$ neutrons, the only explanation for a low-lying $9 / 2^{+}$isomer would be the $9 / 2^{+}[404]$ neutron configuration. Such an excitation has been observed recently in ${ }^{97} \mathrm{Sr},{ }^{99} \mathrm{Zr}$, and ${ }^{101} \mathrm{Sr}[23-25]$ but it is not expected at the Fermi surface in ${ }^{115} \mathrm{Pd}$. Moreover, its properties (strongly deformed bands on top of it) are different from what one sees in ${ }^{115} \mathrm{Pd}$;

iii) there is no crossover $(38.8+48.6) \mathrm{keV}$ transition in the spectra.

Considering all these arguments we conclude that both, the $38.8 \mathrm{keV}$ and the $48.6 \mathrm{keV}$ transitions are of a M1+E2 multipolarity and it is likely that they depopulate the $11 / 2^{-}$level in parallel cascades. Consequently, two new levels are defined below the $11 / 2^{-}$band head, which does not correspond anymore to the $50 \mathrm{~s}$ isomer reported in ${ }^{115} \mathrm{Pd}[21]$. It is also unlikely that the higher of the two new levels corresponds to the $50 \mathrm{~s}$ isomer because it could decay to the lower level by a M1+E2 transition. We propose that the lower level, located $48.6 \mathrm{keV}$ below the $11 / 2^{-}$ band head, as shown in Fig. 2 in the partial decay scheme of ${ }^{115} \mathrm{Pd}$ obtained in this work, corresponds to the isomer with half-life of 50 seconds reported at $89.3 \mathrm{keV}$ [21].

Coincidence relations presented in Fig.2 are consistent with the existence of an isomer at $89.1 \mathrm{keV}$. The 89.1 $\mathrm{keV}$ level, shown in Fig.2 is fed by the $164.6 \mathrm{keV}$ line (observed already in Refs.[14,15]) and a new $265.6 \mathrm{keV}$ line seen in our data. Since no decay of the $89.1 \mathrm{keV}$ level is observed in our data, this level must have a long halflife. The excitation energy of this level is very close to the $89.3 \mathrm{keV}$ excitation energy of the isomer measured in [21]. We propose that the $89.1 \mathrm{keV}$ level corresponds to the 50

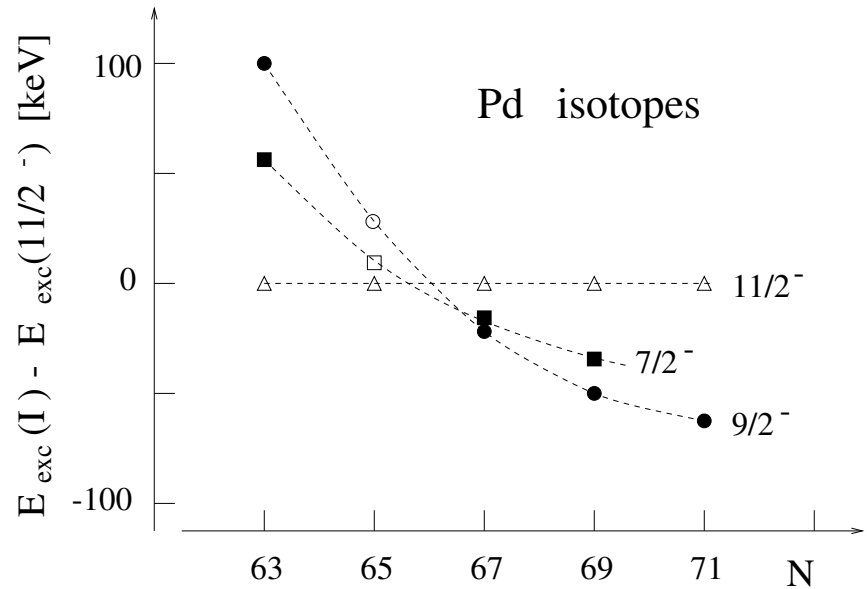

Fig. 3. The position of the $7 / 2^{-}$and $9 / 2^{-}$excitations relative to the $11 / 2^{-}$level in odd- $\mathrm{N}$ palladium isotopes. The data at $\mathrm{N}=69$ and 71 are from this work and at other $\mathrm{N}$ values from Refs.[14-16,26]. Open square and open circle mark values expected for the $7 / 2^{-}$and $9 / 2^{-}$levels in ${ }^{111} \mathrm{Pd}$, respectively. Dashed lines are drawn to guide the eye.

seconds isomer while the $11 / 2^{-}$band head is now located at $137.7 \mathrm{keV}$.

To propose spins for the two new levels we inspect excitation energies of negative-parity levels in the neighbouring, odd-N Pd isotopes. Energies of the $7 / 2^{-}$and $9 / 2^{-}$ levels in ${ }^{109} \mathrm{Pd}[16]$ and ${ }^{113} \mathrm{Pd}[14,15,26]$ are shown in Fig.3, relative to the energy of the $11 / 2^{-}$band head in these nuclei. In Fig.3 we also show relative energies of the two new levels in ${ }^{115} \mathrm{Pd}_{69}$, seen in this work. The $89.1 \mathrm{keV}$ level follows the trend of $9 / 2^{-}$excitations and the 99.4 $\mathrm{keV}$ level (proposed $38.3 \mathrm{keV}$ below the $11 / 2^{-}$level but not displayed in Fig.2) fits the trend of $7 / 2^{-}$excitations.

The above result has implications for the spin of the ground state in ${ }^{115} \mathrm{Pd}$. With the $9 / 2^{-}$spin of the 89.1 $\mathrm{keV}$ isomer and the isomeric transition of a stretched $\mathrm{E} 3$ multipolarity, the spin and parity of the ground state of ${ }^{115} \mathrm{Pd}$ is $\mathrm{I}^{\pi}=3 / 2^{+}$, instead of $5 / 2^{+}$reported in [11-15]. Let us mention that the $3 / 2^{+}$spin assignment to the ground state in ${ }^{115} \mathrm{Pd}$ was considered already in Refs.[14, 15, 22]. As reported there, the $11 / 2^{-}$spin assignment to the isomer (and, consequently, spin $5 / 2^{+}$for the ground state) was chosen due to the systematics of the $11 / 2^{-}$levels, thought to be isomers in all odd-A Pd isotopes, whereas $\beta^{-}$-decay properties of ${ }^{115} \mathrm{Pd}$ indicated spin $3 / 2^{+}$for the ground state (and, consequently, spin $9 / 2^{-}$for the isomer).

The assignment of $3 / 2^{+}$spin to the ground state and the $9 / 2^{-}$to the $89.1 \mathrm{keV}$ isomer fixes spins of the 127.9 $\mathrm{keV}$ and $253.7 \mathrm{keV}$ levels at $5 / 2^{+}$and $7 / 2^{+}$, respectively, since the $127.9 \mathrm{keV}$ and $125.8 \mathrm{keV}$ transitions are of M1+E2, $\Delta \mathrm{I} \leq 1$ character $[15,27]$ and, consequently, the $164.6 \mathrm{keV}$ transition must be a stretched E1 (M2 multipolarity is unlikely, considering prompt character of this transition). One can also limit the spin of the $354.7 \mathrm{keV}$ level to $7 / 2^{+}$ or $9 / 2^{+}$due to the presence of the $265.6 \mathrm{keV}$ transition of prompt character. The $9 / 2^{+}$spin value is preferred since 


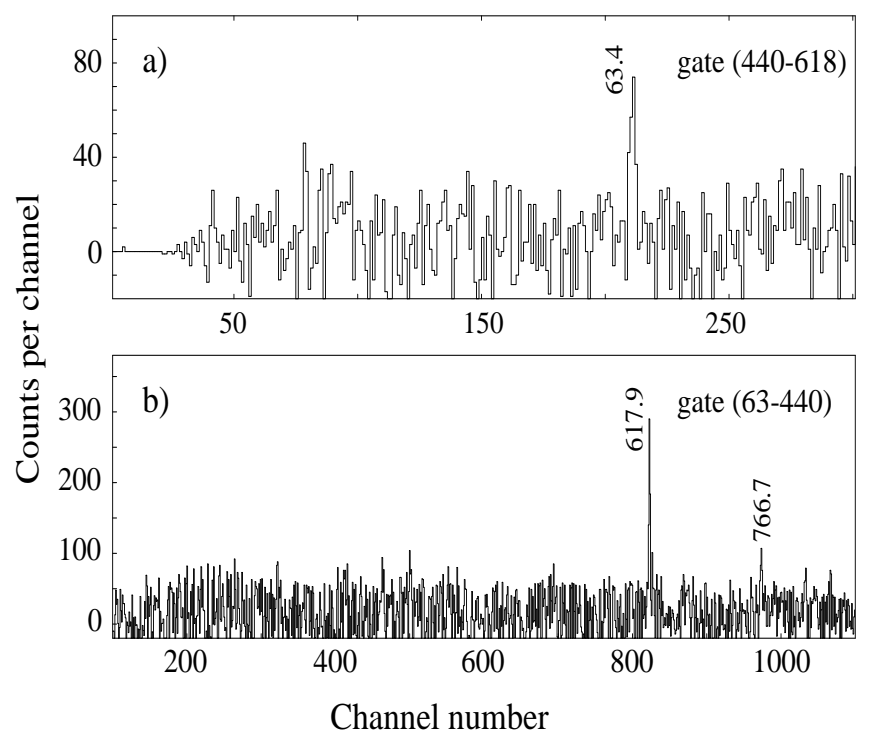

Fig. 4. Coincidence spectra double-gated on lines in ${ }^{117} \mathrm{Pd}$. Transition energies and gates are given in $\mathrm{keV}$.

otherwise, the band on top of the $354.7 \mathrm{keV}$ level becomes rather non-yrast, while it is commonly observed that spontaneous fission populates preferably yrast states.

This last argument helps to discriminate against another possibility, namely that the $38.8 \mathrm{keV}$ and the 48.6 $\mathrm{keV}$ transitions are in a cascade. Although in such a version the observed properties of both transitions are easier to explain, it implies spin $7 / 2^{-}$for the isomer and, consequently, spin $1 / 2^{+}$for the ground-state of ${ }^{115} \mathrm{Pd}$. This in turn fixes spins and parities of the $127.9 \mathrm{keV}$ and the $253.7 \mathrm{keV}$ levels to $3 / 2^{+}$and $5 / 2^{+}$, respectively. Consequently, the spin of the $354.7 \mathrm{keV}$ level should be $5 / 2^{+}$ or $7 / 2^{+}$, making the band on top of the $354.7 \mathrm{keV}$ level rather non-yrast, while it is populated stronger that the yrast, negative-parity band. Therefore, the population argument supports spin $3 / 2^{+}$for the ground state and spin $9 / 2^{-}$for the isomer.

It has to be remarked, however, that the $1 / 2^{+}$possibility will need further studies. The $1 / 2^{+}$orbital of the $d_{3 / 2}$ parentage is positioned close to the Fermi surface at $\mathrm{N}=69$, as has been shown in our recent study of ${ }^{113} \mathrm{Ru}[26]$, the isotone of ${ }^{115} \mathrm{Pd}$, where the $\mathrm{T}_{1 / 2}=0.5 \mathrm{~s}$ isomer [28] has been assigned spin $7 / 2^{-}$and the ground state spin $1 / 2^{+}[26]$. Moreover, spin $1 / 2^{+}$was considered for the ground state in ${ }^{115} \mathrm{Pd}$ already in Ref.[22], based on the observed feeding of the ground state of ${ }^{115} \mathrm{Pd}$ in $\beta^{-}$-decay of the $7 / 2^{+}$ ground state of ${ }^{115} \mathrm{Rh}$. Finally, the $1 / 2^{+}$level is reported at low energies in the neighbouring odd- $\mathrm{N}$ Pd isotopes[14, 15]. Considering all this, the $1 / 2^{+}$ground state in ${ }^{115} \mathrm{Pd}$ would not be unusual.

The ${ }^{117} \mathrm{Pd}$ nucleus was studied before in spontaneous fission [11]. In Fig. 4a we show a low-energy part of the spectrum, double gated on the $440 \mathrm{keV}$ and $618 \mathrm{keV}$ lines from the negative-parity band in ${ }^{117} \mathrm{Pd}$, reported in Ref.[11]. In the spectrum one can see a new line at $63.4 \mathrm{keV}$. In a

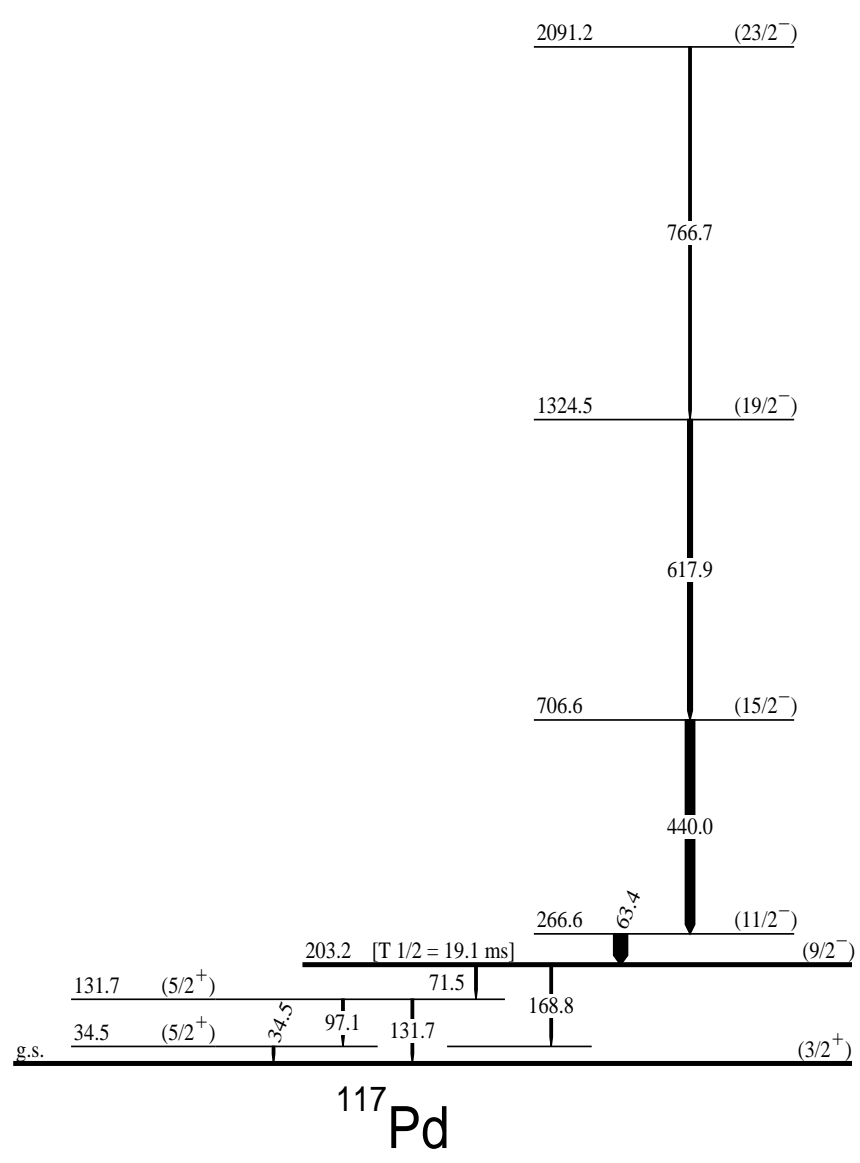

Fig. 5. Partial decay scheme of ${ }^{117} \mathrm{Pd}$ as proposed in this work.

spectrum double-gated on the $63.4 \mathrm{keV}$ and the $440.0 \mathrm{keV}$ lines, shown in Fig. $4 \mathrm{~b}$, there are $617.9 \mathrm{keV}$ and $766.7 \mathrm{keV}$ lines of the negative-parity band in ${ }^{117} \mathrm{Pd}$. Intensities seen in gated spectra suggest that the $63.4 \mathrm{keV}$ transition is located in the negative-parity band below the $440.0 \mathrm{keV}$ line. The $63.4 \mathrm{keV}$ transition defines thus a new level below the $11 / 2^{-}$band head, as shown in Fig.5 in the partial level scheme of ${ }^{117} \mathrm{Pd}$ obtained in this work. We propose that the new level corresponds to the $203.2 \mathrm{keV}$ isomer with the half-life of $19.1 \mathrm{~ms}$, reported in Ref.[27]. Consequently, the $11 / 2^{-}$band head is now located at 266.6 $\mathrm{keV}$.

In Fig.3 we show the position of the new $9 / 2^{-}$level in ${ }^{117} \mathrm{Pd}$, relative to the $11 / 2^{-}$band head. The level fits well the trend of $9 / 2^{-}$excitations in odd-N Pd isotopes. We note, that the regularity of this picture allows the estimate of the, yet unknown, positions of the $7 / 2^{-}$and $9 / 2^{-}$levels in ${ }^{111} \mathrm{Pd}$ expected about $10 \mathrm{keV}$ and $30 \mathrm{keV}$ above the $11 / 2^{-}, 172 \mathrm{keV}$ isomer $[15,16]$, respectively. Experimental identification of these two levels would add credibility to the proposed picture.

The $9 / 2^{-}$spin assignment to the isomer in ${ }^{117} \mathrm{Pd}$ has implications for the spin of the ground state and the 34.5 $\mathrm{keV}$ and $131.7 \mathrm{keV}$ levels [27] in this nucleus. In Ref.[27] it was found that the $71.5 \mathrm{keV}$ and $168.8 \mathrm{keV}$ isomeric transitions are of a stretched M2 character. Consequently, 
considering that the spin of the $203.2 \mathrm{keV}$ isomeric level is $9 / 2^{-}$, we propose that the spin and parity of both, the $34.5 \mathrm{keV}$ and the $131.7 \mathrm{keV}$ levels are $\mathrm{I}^{\pi}=5 / 2^{+}$, instead of $7 / 2^{+}$reported previously [27]. The multipolarity of the $34.5 \mathrm{keV}$ ground-state transition in ${ }^{117} \mathrm{Pd}$ was reported as $\mathrm{M} 1, \Delta \mathrm{I}=1$ [27], because of the non-observation of a M2 decay of the $203.2 \mathrm{keV}$ isomer to the ground state. Considering this and the new $\mathrm{I}^{\pi}=5 / 2^{+}$spin of the 34.5 $\mathrm{keV}$ level, we propose that the ground-state spin of ${ }^{117} \mathrm{Pd}$ is $\mathrm{I}^{\pi}=3 / 2^{+}$, instead of $\mathrm{I}^{\pi}=5 / 2^{+}[27]$.

The new spin and parity assignments in ${ }^{115} \mathrm{Pd}$ and ${ }^{117} \mathrm{Pd}$, proposed in this work, are consistent with the available Nilsson excitations at neutron number $\mathrm{N}>69$. The change of palladium ground-state spin from $5 / 2^{+}$at $\mathrm{N} \leq 67$ to $3 / 2^{+}$at $\mathrm{N}>67$ is what one expects. Inspecting the scheme of single-neutron levels calculated for this region (see e.g. [10]), one finds that at moderate prolate deformation of $\beta_{2} \approx 0.2$, the Fermi level approaches both the $9 / 2^{-}$[514] orbital of the $\nu \mathrm{h}_{11 / 2}$ shell as well as the $3 / 2^{+}$[402] orbital of the $\nu \mathrm{d}_{3 / 2}$ shell. The latter configuration has been recently observed in the ${ }^{111} \mathrm{Ru}$ nucleus [29,30], which supports its presence at the Fermi level around $\mathrm{N}=69$. The new results improve the overall agreement between the experimental data and the calculated pattern of singleneutron levels in odd-N Pd. In this picture both, positiveand negative-parity excitations in odd-N Pd are due to a valence-neutron levels in a moderately-deformed, prolateshaped potential.

Summarizing, we conclude that the available data indicate spins of the $89.1 \mathrm{keV}$ and $203.2 \mathrm{keV}$ isomers in ${ }^{115} \mathrm{Pd}$ and ${ }^{117} \mathrm{Pd}$, respectively, lower than $11 / 2^{-}$, reported previously. Consequently, spins of the ground state in both nuclei are lower than $5 / 2^{+}$. The new data indicate, as a likely solution, spin $9 / 2^{-}$for isomers and $3 / 2^{+}$for ground states in both nuclei. In case of ${ }^{115} \mathrm{Pd}$ both spins may be lowered by one unit, if the future studies would show that the $38.8 \mathrm{keV}$ transition is in cascade with the 48.6 $\mathrm{keV}$ transition in this nucleus. Further detailed studies of neutron-rich odd-N palladium isotopes are of high interest, in order to verify the new spin assignments proposed in this work.

This work was partially supported by the French-Polish IN2P3-KBN collaboration No. 01-100, by the UK EPSRC under grant no. GRH71161 and by the US Dept. of Energy under contract No. W-31-109-ENG-38. The authors are indebted for the use of ${ }^{248} \mathrm{Cm}$ to the Office of Basic Energy Sciences, US Dept. of En., through the transplutonium element production facilities at the Oak Ridge National Laboratory.

\section{References}

1. M.A.C. Hotchkis et al., Nucl. Phys A 530, 111 (1991)

2. J. Skalski, P.-H. Heenen and P. Bonche, Nucl. Phys. A 559, 221 (1993)

3. G. Lhersonneau et al., Phys. Rev. C49, 1379 (1994)

4. W. Urban et al., Nucl. Phys A 689, 605 (2001)

5. M.A.C. Hotchkis et al., Phys.Rev.Lett 64, 3123 (1990)
6. G. Lhersonneau et al., Phys. Rev. C 51, 1211 (1995)

7. J.L Durell, T..J. Armstrong, W.Urban, Acta Phys. Pol. B 34, 2277 (2003)

8. A.G. Smith et al., Phys. Lett. B 591, 55 (2004)

9. J.L Durell, T.J. Armstrong, W.Urban, Eur. Phys. J. A 20, $97(2004)$

10. F.R. Xu, P.M. Walker, R. Wyss, Phys. Rev. C 65, 021303(R) (2002)

11. X.Q. Zhang et al., Phys. Rev. C 61, 014305 (1999)

12. M. Houry et al., Eur. Phys. J. A 6, 43 (1999)

13. R. Krücken et al., Phys. Rev. C 60, 031302 (1999)

14. H. Penttilä, PhD Thesis, Dept. of Physics, University of Jyväskylä, Research Report No. 1/1992

15. H. Penttilä et al., Nucl. Phys. A 561, 416 (1993)

16. T. Kutsarova et al., Phys. Rev. C 58, 1966 (1998)

17. P.J. Nolan, F.A. Beck and D.B. Fossan, Annu. Rev. Nuc. Part. Sci. 44, 561 (1994)

18. W. Urban et al., Z. Phys. A 358, 145 (1997)

19. T. Rząca-Urban et al., Phys. Lett. B 348 (1995) 336

20. W. Urban et al., Phys. Rev. C 61, 41301(R) (2000)

21. B. Fogelberg et al., Z. Phys. A 337, 251 (1990)

22. J. Äystö et al., Phys. Lett. B 201, 211 (1988)

23. W. Urban et al., Eur. Phys. J A 16, 11 (2003)

24. J.K. Hwang et al., Phys. Rev. C 67, 054304 (2003)

25. W. Urban et al., Eur. Phys. J. A, 2004, in print

26. J. Kurpeta et al., to be published

27. H. Penttilä et al., Phys. Rev. C 44, R935 (1991)

28. J. Kurpeta et al., Eur. Phys. J A 2, 241 (1998)

29. W. Urban et al., Eur. Phys. J. A, 2004 in print

30. Ch. Droste et al., Eur. Phys. J. A, 2004, in print 